\title{
Miteinander und aneinander wachsen
}

\author{
Wie können Führungskräfte mit ihren Mitarbeitern anspruchsvolle Aufgaben \\ bewältigen, ohne sich selbst und andere dabei zu verschleissen?
}

Serkan Yildiz, unter Mitwirkung von Walter Kromm

Walter Kromm hat das Buch «Unternehmensressource Gesundheit» herausgegeben. Weitere Einzelheiten unter: www.walter-kromm.de

Korrespondenz: Serkan Yildiz Speakers Excellence Adlerstraße 41 D-70199 Stuttgart Tel. 004971175858478
Auf den ersten Blick mag es überraschend sein, wenn ein Arzt sich mit dem Thema Unternehmensführung beschäftigt. Der Mediziner und Master of Public Health Dr. Walter Kromm ${ }^{1}$ arbeitet und forscht seit vielen Jahren an der «Nahtstelle» zwischen Gesundheit und Unternehmensführung. Die Forschungsergebnisse von Dr. Kromm zeigen, dass sich gesunde und leistungsfähige Menschen von ihren gesundheitlich belasteten und demotivierten Kollegen deutlich darin unterscheiden, wie sie die Unternehmenskultur in ihrem Betrieb beschreiben. Als «Hauptverantwortlichen» für den Gesundheitszustand der im Unternehmen agierenden Leistungsträger identifiziert Dr. Kromm die Qualität der Führung.

Unternehmen sollten deshalb die Perspektive wechseln: Es geht nicht in erster Linie darum, etwas «Gesundes» zu unternehmen oder sich mit Krankenstatistiken zu befassen. Entscheidend sei die «Qualität der Anwesenheit» der Mitarbeiter und der Grad der Widerstandsfähigkeit gegen potentiell gesundheitsgefährdende Einflüsse. Gesundheit im Unternehmen hat also weniger mit Sport-, Ernährungsund Abnehmprogrammen als mit Führung zu tun! Die Unternehmensleitung muss sich deshalb der Frage widmen, wie Führungskräfte mit ihren Mitarbeitern anspruchsvolle Aufgaben bewältigen, ohne sich selbst und andere dabei zu verschleissen.

Um das herauszufinden, hat Dr. Kromm mehrere Studien initiiert. Im Rahmen des Forums Leadership and Health bei der Schweizer UBS wurden die ersten Forschungsergebnisse vorgestellt. An dem Forum wirkten mehr als 120 Wissenschaftler und Führungskräfte aus Deutschland und der Schweiz mit.
Die Ergebnisse der Studien von Walter Kromm widersprechen jedoch dieser Annahme: Führungskräfte erleben zwar einen deutlich höheren «workload» als die Allgemeinbevölkerung, allerdings führen diese höheren Anforderungen nicht, wie lange angenommen, automatisch auch zu mehr körperlichen und psychovegetativen Beschwerden. Vielmehr hängt das Fehlen von psychosozialen Schutzfaktoren mit dem Auftreten von gesundheitlichen Beschwerden zusammen. Leistungsorientierte Menschen mit gesundheitlichen Beeinträchtigungen leiden vermehrt unter chronischem Stress aufgrund eines Mangels an Bedürfnisbefriedigung.

\section{Welche Bedürfnisse werden am stärksten frustriert?}

- Bedürfnis nach Anerkennung, Achtung und Wertschätzung (Selbstwerterhöhung);

- Bedürfnis nach Sicherheit und verlässlichen, tragfähigen Beziehungen (Bindungsbedürfnis);

- Bedürfnis nach Entfaltung und Gestaltungslust (Wachstumsbedürfnis);

- Bedürfnis nach Erholung, Entspannung, Zeit und Musse.

All diese Bedürfnisse sind evolutionär angelegt und nicht verhandelbar. Ihre Befriedigung ist elementar, um gesund bleiben zu können. Sie lassen sich auf Dauer durch materielle oder berufliche Erfolgserlebnisse nicht ausgleichen. Bei der neuesten Studie «Der ideale Chef» wurden die Studienteilnehmer gebeten, sich vorzustellen, welche Bedürfnisse ein idealer Chef ihnen gegenüber befriedigen würde. Das onto-

\section{Achtsame und wertschätzende Atmosphäre sorgt dafür, dass Arbeitnehmer sich wechselseitig die Energiedepots auffüllen.}

\section{Ist es ungesund, beruflich ambitioniert zu sein und viel zu arbeiten?}

Lange Zeit hielt sich der Glaube, dass chronischer Stress hauptsächlich aus zu hohen Anforderungen resultiert. Die Definition der «Managerkrankheit» besagt, dass in erster Linie von einem hohen «workload», also hoher Arbeitsbelastung, hoher Verantwortung für Mitarbeiter und hohem Erfolgsdruck ein Risiko für die Gesundheit ausgeht. genetisch früheste und neurobiologisch am tiefsten verankerte Bedürfnis nach stabilen, verlässlichen und ermutigenden Beziehungen wurde dabei am häufigsten genannt.

Einer der wichtigsten Erkenntnisse von Kromms Forschungsprojekten ist, dass nicht die beruflichen und privaten Anforderungen per se, sondern das Ausmass der Frustration der oben erwähnten Bedürfnisse den grössten Einfluss auf die Gesundheit und 
das Wohlbefinden der im Unternehmen agierenden Menschen hat. Menschen, bei denen es an den oben erwähnten Schutzfaktoren mangelt, werden gesundheitlich stark von hohen Arbeitsanforderungen getroffen. Im Gegensatz dazu erleben Menschen, die hohen Erwartungen gerecht werden müssen, dann die wenigsten gesundheitlichen Beeinträchtigungen, wenn ihnen diese gesundheitsförderlichen Ressourcen nicht vorenthalten werden. Eine achtsame, unterstützende und wertschätzende Unternehmenskultur hilft somit Leistungsträgern, die potentiell gesundheitsgefährdenden Effekte hoher Belastungen abzupuffern.

\section{Vorgesetzte bezeichnet man als Führungs- kraft, weil von ihnen Kraft ausgehen soll}

Das wichtigste Potential eines Unternehmens sind all jene Führungskräfte, denen es gelingt, die Arbeitsbedingungen in ihrem Verantwortungsbereich trotz hoher Anforderungen - möglichst gesundheitsgerecht zu gestalten und somit die Widerstandskraft der Mitarbeiter gegenüber potentiell gesundheitsbedrohenden Einflüssen zu stärken. Führungskräften mit derartigen Fähigkeiten gelingt es dann auch besser, gesundheitsförderliche Ressourcen von anderen zu erhalten. Eine achtsame und wertschätzende Atmosphäre sorgt dafür, dass die im Unternehmen agierenden Menschen sich wechselseitig die Energiedepots auffüllen. Somit ist es möglich, dass Führungskräfte mit ihren Mitarbeitern anspruchsvolle Aufgaben bewältigen können, ohne sich selbst und andere dabei zu verschleissen.

Je höher die Ressourcenausstattung der Mitarbeiter ist, umso leichter ist es, bei ihnen die Lust zu wecken, sich einzubringen, mitzudenken, mitzugestalten und ihr kreatives Potential zu entfalten. Langfristig gesunde und motivierte Mitarbeiter und

\section{Corporate Health Convention}

Europäische Fachmesse für betriebliche Gesundheitsförderung und Demografie 9. und 10. April 2013 in der Messe Zürich, Halle 3
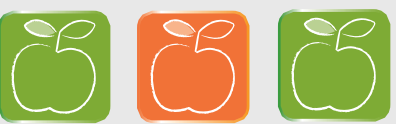

CORPORATE

HEALTH CONVENTION

Walter Kromm an der Corporate Health Convention: Am 10. April ist Walter Kromm mit seinem Vortrag «Miteinander und aneinander wachsen» als Keynote Speaker an der Corporate Health Convention zu Gast. Sein Vortrag beginnt um 14.30 Uhr im Praxisforum 2.

Die Corporate Health Convention ist die Europäische Fachmesse für betriebliche Gesundheitsförderung und Demografie und findet nach Stationen in Bern und Basel am 9. und 10. April 2013 zum ersten Mal in Zürich statt. Die Fachmesse verspricht Informationen, Austausch und Netzwerke im Namen der Gesundheit und präsentiert Produkte, Dienstleistungen und Strate-

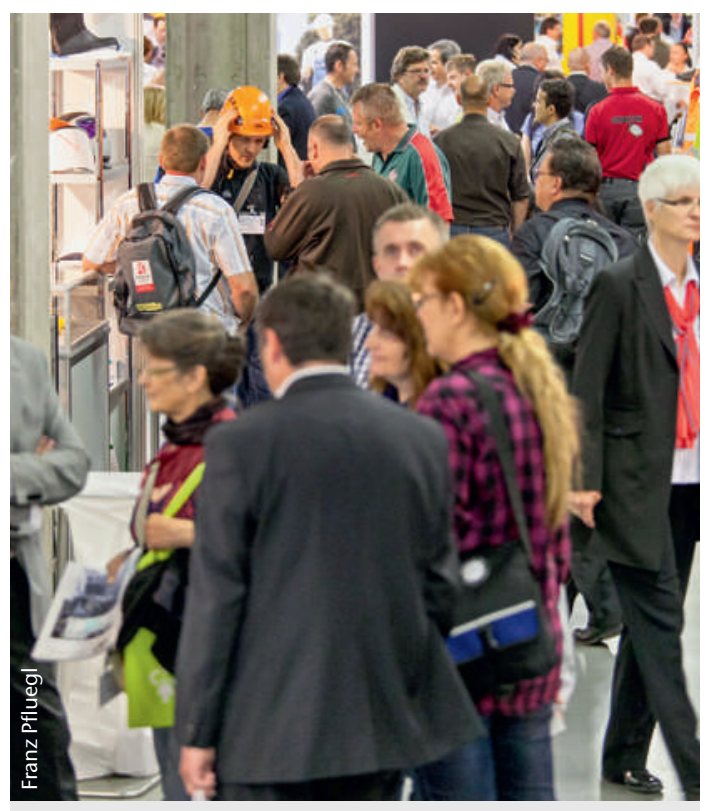

Die Messe «Corporate Health Convention» informiert über betriebliche Gesundheitsförderung sowie altersund alternsgerechte Arbeitsbedingungen.

der langfristige betriebswirtschaftliche Erfolg eines Unternehmens sind zwei Seiten der gleichen Medaille - und beide werden zentral beeinflusst von den Bestimmungsfaktoren guter Führung.

\section{Fazit}

Das Geheimnis guter Führung ist die Kunst, miteinander und aneinander zu wachsen durch Ressourcenaustausch. Führungskräfte, die sich dieser Thematik widmen, werden überrascht sein, welche Produktivitätsreserven und wie viel Lebensqualität in ihren Unternehmen schlummern.

gien, damit Unternehmen sich stärker um das Wohl ihrer Belegschaft kümmern können. Dabei finden die Themenbereiche Prävention, Ergonomie, betriebliche Gesundheitsförderung und alters- und alternsgerechte Arbeitsbedingungen viel Raum im Messegeschehen. Neben den Ausstellerständen und den Vorträgen in den Praxisforen, darunter vier hochkarätige Keynotes, fordern einige Programmformate die Fachbesucher direkt zum Mitmachen und Aktivwerden auf. Die Fachmesse richtet sich an Führungskräfte, HR-Verantwortliche, Gesundheitsmanager und all jene, die in der gesundheitsunterstützenden Ausrichtung von Arbeitsbedingungen und Arbeitsplätzen und in der wertschätzenden Behandlung von Mitarbeitern die Grundlage für unternehmerischen Erfolg sehen.

Anmeldung und weitere Informationen: www.corporate-health-convention.ch.

Die Schweizerische Ärztezeitung ist Medienpartner der Corporate Health Convention. 